\title{
Request for Research Proposals 2011 CAG/CIHR/Industry Research Program
}

\footnotetext{
The CAG/CIHR/Industry Research Program continues to be a very successful and highly sought after funding avenue. To date, more than 165 researchers have been supported by this collaborative program. This amounts to more than $\$ 16$ million worth of the highest quality research in the field of gastroenterology in Canada. Many of the researchers supported by this program have been appointed in clinical and/or academic institutions within Canada, and have secured competitive independent research funding from the CIHR and other agencies.
}

The following funding opportunities are open for the 2011 CAG/CIHR/Industry Research Program:

CAG/CIHR/Industry Fellowships

CAG/CIHR/CCFC IBD Fellowships: two-year term (3)

CAG/CIHR/Abbott IBD Fellowship Award: two-year term (1)

CAG/CIHR/Ferring IBD Fellowship: two-year term (1)

Fellowship applications MUST be submitted directly to CIHR by October 1, 2010.

CAG/CIHR/Industry New Investigator Salary Award

CAG/CIHR/CCFC New Investigator Salary Award: five-year term (1)

New Investigator applications MUST be submitted directly to CIHR by September 15, 2010.

CAG/CIHR/Industry Operating Grants

CAG/CIHR/Abbott IBD Operating Grant: two-year term (1)

CAG/CIHR/AstraZeneca Upper Gastrointestinal Operating Grant: two-year term (1)

CAG/CIHR/Merck IBD Operating Grant: two-year term (1)

CAG/CIHR/Olympus Endoscopic Research Operating Grant: two-year term (1)

Operating Grant applications MUST be submitted directly to CIHR by November 1, 2010.

These opportunities, as well as links to the CIHR website for more details and application forms, are outlined at http://www.cag-acg.org/research.aspx.
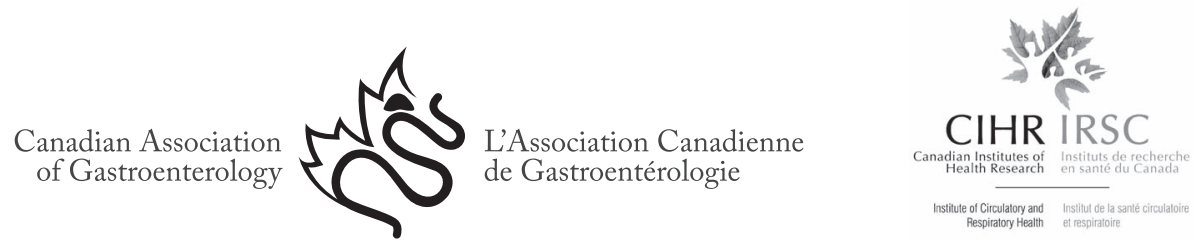


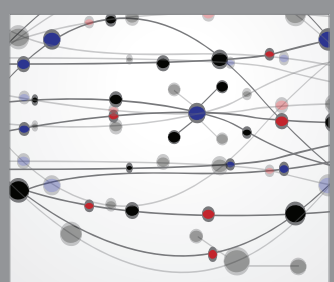

The Scientific World Journal
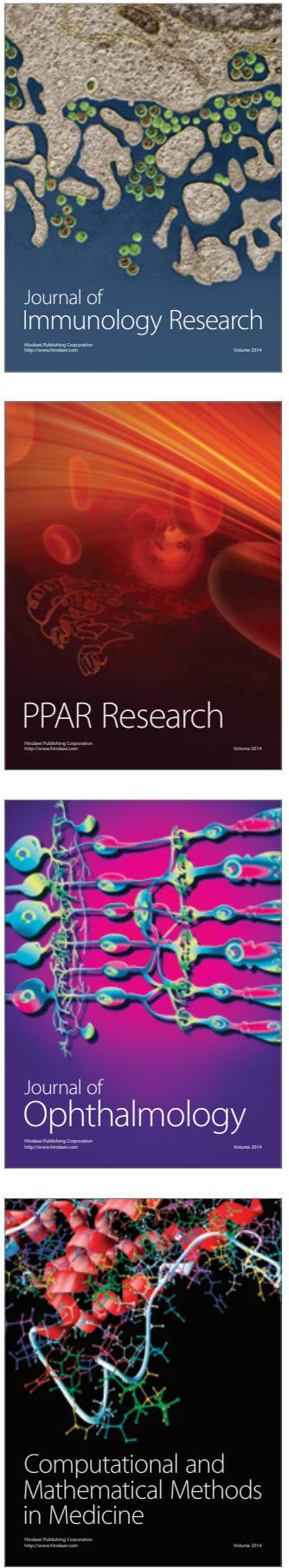

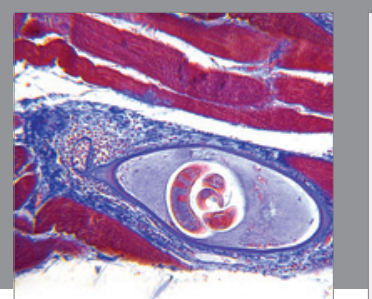

Gastroenterology Research and Practice

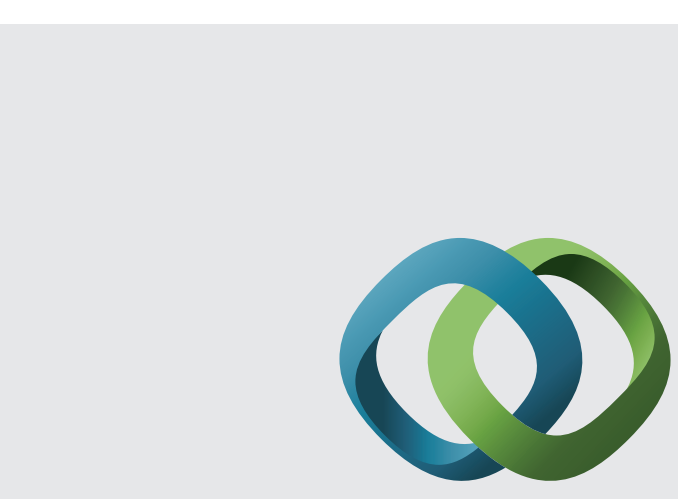

\section{Hindawi}

Submit your manuscripts at

http://www.hindawi.com
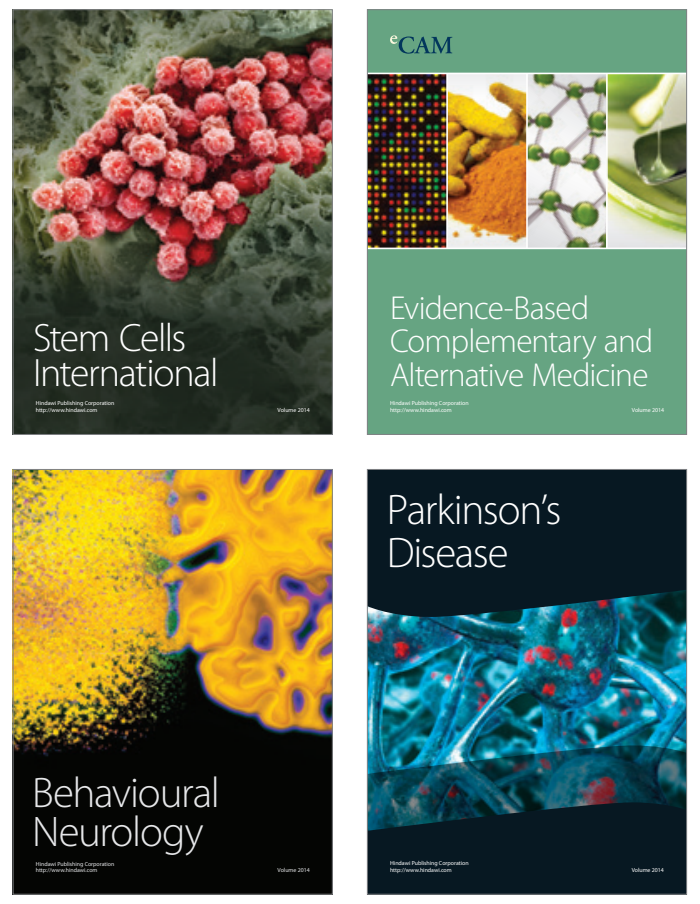
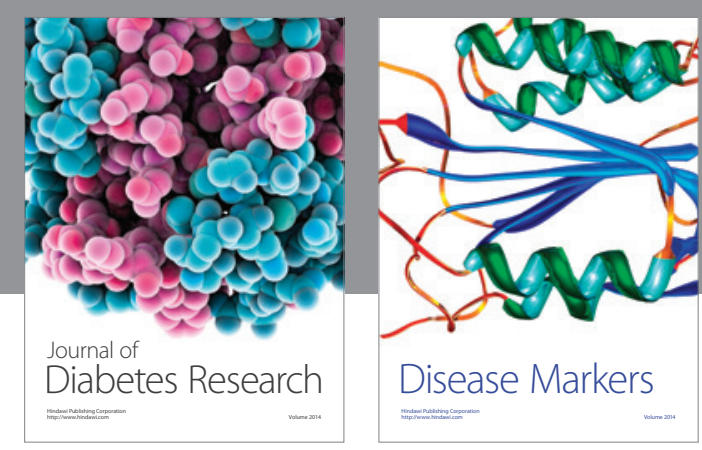

Disease Markers
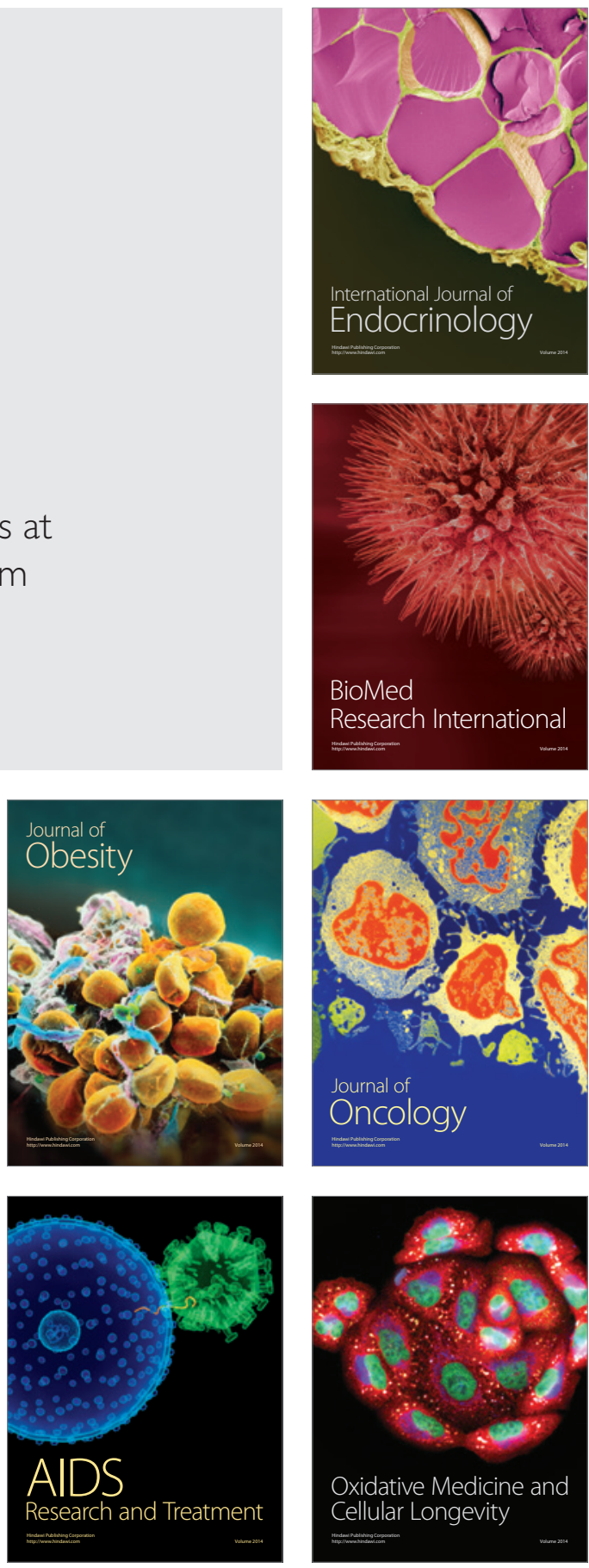